\title{
Article \\ An Adaptive Approach for Impulsive Formation Maintenance Relevant to Distributed SAR Missions
}

\author{
Salvatore Sarno ${ }^{1, *(D)}$, Mariano Iervolino $^{2}$ and Giancarmine Fasano ${ }^{2, *}$ \\ 1 Department of Engineering, University of Campania "L. Vanvitelli", Via Roma 29, 81031 Aversa, Italy \\ 2 Department of Industrial Engineering, University of Napoli “Federico II", P. leTecchio 80, 80125 Napoli, Italy; \\ marianoiervo@gmail.com \\ * Correspondence: salvatore.sarno@unicampania.it (S.S.); giancarmine.fasano@unina.it (G.F.)
}

Citation: Sarno, S.; Iervolino, M.; Fasano, G. An Adaptive Approach for Impulsive Formation Maintenance Relevant to Distributed SAR Missions Aerospace 2022, 9, 142. https:// doi.org/10.3390/aerospace 9030142

Academic Editor: Dario Modenini

Received: 19 January 2022

Accepted: 2 March 2022

Published: 6 March 2022

Publisher's Note: MDPI stays neutral with regard to jurisdictional claims in published maps and institutional affiliations.

Copyright: (c) 2022 by the authors. Licensee MDPI, Basel, Switzerland. This article is an open access article distributed under the terms and conditions of the Creative Commons Attribution (CC BY) license (https:// creativecommons.org/licenses/by/ $4.0 /)$.

\begin{abstract}
This paper proposes an approach for impulsive formation maintenance tailored to distributed synthetic aperture radar, i.e., a spaceborne system composed by several antennas working together to provide enhanced remote sensing capabilities. The analyzed configuration is designed to guarantee the presence of a safety tube surrounding each satellite as the dynamics evolve. Formation requirements are related to general constraints on the acceptable along-track and radial/cross-track separations. The paper introduces an adaptive maintenance logic which fulfills these constraints. Specifically, the formation is adaptively redesigned around the chief every time geometry constraints are violated by means of a procedure developed by the authors in previous works and based on relative orbit parameters. Once these parameters are defined, the optimal impulsive burns required for orbit transfer are computed using state-of-the-art approaches. Performance in terms of delta- $v$ and maneuver frequency is analyzed for a two-spacecraft formation exploiting a simulation environment based on MATLAB and GMAT. In ideal conditions, it is shown that maintenance costs are limited, while close proximity requires fine sensitivity on the applied impulses. A first assessment of the impact of relative navigation and maneuvering execution errors indicates that they play an important role in defining the overall control effort.
\end{abstract}

Keywords: autonomous formation flying; relative GNC; distributed SAR; adaptive formation maintenance; passively safe configurations

\section{Introduction}

It is widely agreed that many upcoming space missions will rely on distributed systems exploiting co-flying, cooperating platforms to replace current monolithic systems, and to implement missions otherwise impossible (e.g., those requiring very large sensor apertures) or extremely complex [1].

Unleashing the potential of such evolution requires the addressing of challenges both at a payload and at a system level, which are closely related to each other. Fractionating the payload on multiple space platforms requires the development of processing approaches that account for (and take advantage from) the spatial diversity of measurements. At a system level, the necessity that multiple different spacecraft act as a single entity leads to proper architectures and algorithmic approaches for relative trajectory design, relative navigation and control, inter-satellite communication, and distributed/centralized decision making. In fact, the development of distributed space systems is consistent with other major technological trends in the space field, such as increased autonomy and enhanced capabilities of small satellites.

Distributed space mission concepts have been proposed in fields such as planetary and astronomical applications [2], and Earth observation [1]. Within the latter category, the concept of distributed synthetic aperture radar (DSAR) has received increasing attention during the last years [3,4]. DSAR is defined as a synthetic aperture radar in which the signal emitted by the transmitter and scattered from the area of interest is not collected by a single receiver, but by many conveniently distributed formation flying receivers. The concept of distributed aperture is a generalization of the conventional synthetic aperture 
radar (SAR) principle [5], and of standard interferometric SAR (InSAR) techniques [6], towards a flexible system able to implement a wide range of different working modes.

As these different working modes require specific observation geometries, formation flying represents a key technology for DSAR implementation, and aspects such as relative navigation accuracy, control accuracy, and $\Delta v$ expense are key factors for mission design and feasibility.

Within this framework, this paper proposes a formation maintenance approach that is tailored to DSAR applications. In particular, working modes requiring linear or quasilinear formation geometries are considered. These do not need a regular separation among receivers but impose more general constraints on relative motion. As a main original aspect of the proposed approach, DSAR requirements are translated into three-dimensional constraints that the relative geometry must fulfil, and not into nominal trajectories that must be followed with a given tolerance as it happens in other formation control papers.

The presented approach is adaptive since, when geometric requirements are violated, the formation is redesigned as a function of the actual chief orbital parameters. The design methodology, introduced by the authors in previous studies $[7,8]$, works in the domain of mean orbital parameters and is based on the concept of passively safe ellipses, that are relative configurations conceived to meet both payload and collision risk requirements. Therefore, unlike other contributions in the open literature, the adaptive maintenance strategy is not aimed at preserving either constant differences in orbital parameters or constant relative orbit parameters [9], but at guaranteeing DSAR requirements and safe formation flying. Once the required orbital parameters differences are calculated, control actions are determined using a state-of-the-art approach [9]. Maintenance performance and cost are assessed within a realistic simulation environment that exploits MATLAB and the NASA General Mission Analysis Tool (GMAT), while also accounting for the effects of relative navigation errors and thruster errors.

The paper is organized as follows. Section 2 recalls the geometric requirements relevant to DSAR operations and summarizes the formation design approach and the long-term stability properties of the designed trajectories. The formation maintenance approach is described in Section 3, which also recalls the method to determine control actions and the system organizational architecture that has been assumed. Finally, Section 4 briefly describes the adopted simulation environment and discusses formation maintenance performance in different cases, while also considering moderate relative navigation and maneuvering execution errors.

\section{Relative Geometries for Distributed SAR Applications}

In general, DSAR applications require proper acquisition geometries to be fulfilled by the spacecraft formation. As described in [10], DSAR working modes and applications include: signal-to-noise ratio (SNR) improvement, resolution enhancement (in azimuth and in range), pulse repetition frequency (PRF) reduction (which enables high resolution wide swathHRWS), 3D imaging, ground moving target indication (GMTI), and miscellaneous applications. While some of these applications, such as HRWS [11] and GMTI [12], require along-track observation geometries, i.e., a linear distribution of the receivers, other ones, such as coherent range resolution enhancement [13], exploit relatively-large radial/cross-track baselines.

In this work, the focus is set on radar modes, such as HRWS, which require linear observation geometries and a constrained along-track separation to enable coherent processing of gathered signals. Indeed, a regular separation among the receivers is not strictly necessary, while the baselines need to be known accurately in the post processing phase. Formation design and maintenance are aimed at combining payload requirements with system-level constraints, such as minimization of collision risk, reduction of control effort, and implementation of inter-satellite link architectures with reasonable budgets.

Relative motion design and long-term stability analyses are recalled in the next subsections. Within the paper, relative motion is described in the Hill reference frame of a reference platform (defined as chief, while the other satellites are named deputies) - with $x$ 
axis in the orbit radius direction, $z$ axis parallel to the orbit momentum vector, and $y$ axis completing the right-handed coordinate system.

\subsection{Formation Design: The Safety-Tube Concept}

As stated above, radar processing requires a linear distribution of the receivers and a constrained along-track separation. Assuming operation in the $\mathrm{X}$ band, this constraint is set equal to $500 \mathrm{~m}$ [10]. In order to partially decouple collision risk from along-track dynamics, thus allowing the satellites to drift in the along-track direction, the assumed geometry is not purely linear, and a small radial/cross-track baseline is achieved along the whole orbit. This radial/cross-track separation generates a topographic phase to be compensated by DSAR processing [14]. While research is under way to develop compensation algorithms tailored to DSAR, and to analyze the impact of uncertainties relevant to adopted digital elevation models (DEMs), it is assumed here that the acceptable radial/cross-track baseline is limited to a few tens of meters.

Configurations selected in this paper fall within the class of passively safe ellipses: the relative motion of each satellite (deputy) with respect to the reference platform (chief) never trespasses an imaginary safety tube of radius $d_{x z}$ encompassing the chief itself. This also implies the presence of a minimum radial/cross-track baseline between each couple of deputies. These patterns allow the different spacecraft composing the fractionated antenna to fly very close, but in a passively safe condition. In detail, the differences in orbital parameters for each deputy are retrieved by means of the procedure described in [8], which identifies the differences $\delta$ between deputies and chief semi-major axes $(a)$, eccentricities $(e)$, inclinations $(i)$, right ascensions of the ascending node $(\Omega)$, arguments of perigee $(\omega)$, and mean anomalies $(M)$ moving from the set of design parameters $\left[d_{x z}, A_{x}, \widetilde{z}\right]$ able to characterize the shape of relative motion. The procedure is here recalled for the sake of clarity. More specifically, $A_{x}$ is the amplitude of the radial oscillation and $\widetilde{z}$ the out-of-plane component of relative trajectory when the along-track separation nullifies. The choice of the latter parameter generally decides for $A_{z}$ [8], which is the amplitude of the cross-track oscillation. In the case under consideration, $\widetilde{z}$ is set equal to zero (it means that the out-of-plane baseline is entirely radial when $y=0$ ) and the design set $\left[d_{x z}, A_{x}, \tilde{z}\right]$ turns into $\left[d_{x z}, A_{x}, A_{z}\right]$.

Configurations characterized by a set of parameters so that $A_{x}=A_{z}=d_{x z}$ are analysed in the following sections. Hence, the differences in orbital parameters $[\delta a, \delta e, \delta i, \delta \Omega, \delta \omega, \delta M]$ identifying a "safety-tube" formation around a chief satellite are determined through the pseudo-code in Algorithm 1, which summarizes the design procedure for the fixed cross-track safe ellipses described in [8].

Figure 1 shows a two-spacecraft formation designed by means of the presented approach, where a platform whose features are described in Table 1 has been considered as the reference one. The deputy is a satellite equal to the chief and arranged with respect to it so that it cannot cross a safety tube with radii equal to $20 \mathrm{~m}$.

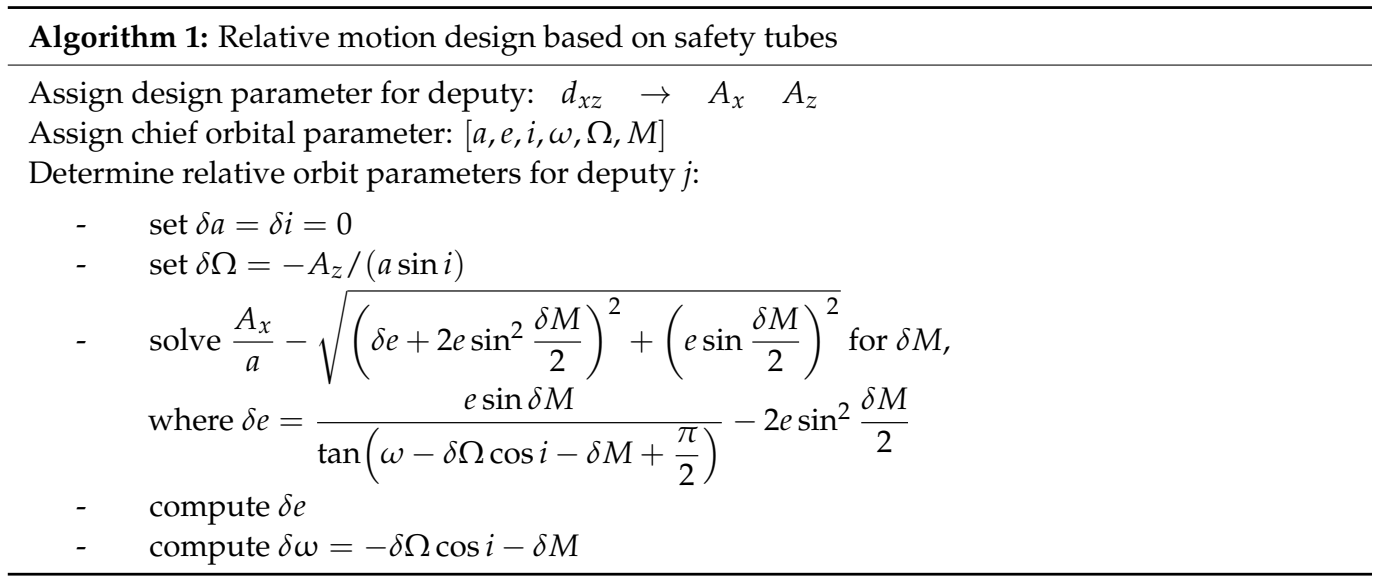


Table 1. Properties of reference satellite.

\begin{tabular}{|c|c|c|c|c|c|}
\hline \multicolumn{6}{|c|}{ Spacecraft Features } \\
\hline Mass (kg) & & Frontal Area $\left(\mathrm{m}^{2}\right)$ & & Ballistic Coef. $\left(\mathrm{kg} / \mathrm{m}^{2}\right)$ & \\
\hline 50 & & 1 & & 22.73 & \\
\hline \multicolumn{6}{|c|}{ Initial mean orbit elements } \\
\hline$a(\mathrm{~km})$ & $e$ & $i\left(^{\circ}\right)$ & $\Omega\left({ }^{\circ}\right)$ & $\omega\left(^{\circ}\right)$ & $M\left({ }^{\circ}\right)$ \\
\hline 6928.1 & 0.001 & 97.59 & 0 & 45 & 0 \\
\hline
\end{tabular}

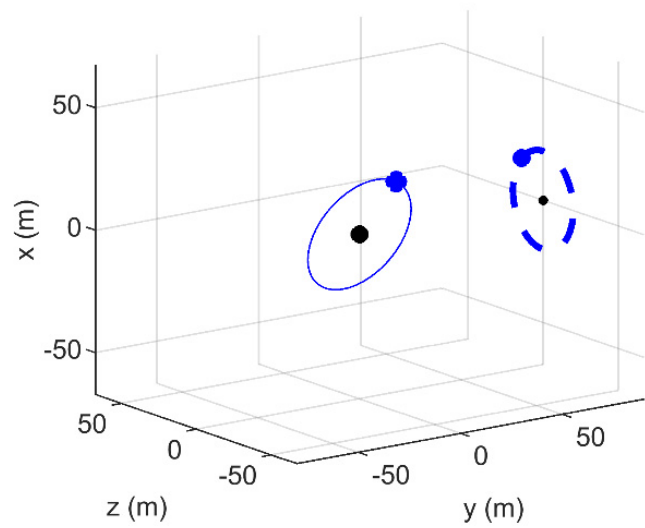

Figure 1. Spacecraft formation based on safety tubes as seen in the Hill reference frame of the chief satellite (black dot). The deputy (blue dot) is arranged to orbit around the chief so that its radial/crosstrack baseline (dashed trajectories in the $x z$ plane) encompasses a safety tube of radii equal to $20 \mathrm{~m}$.

\subsection{Free Evolution of Configurations Based on Safety Tubes}

The method recalled in the previous section allows the formation to be designed according to safety requirements and operational constraints. Nonetheless, perturbation sources that have not been included during its mathematical derivation would be responsible for corruption of nominal geometries. Furthermore, unavoidable errors on initial conditions of relative motion, and residual differential effects, make formation maintenance necessary.

Before analyzing the proposed formation maintenance approach, it is useful to analyze the free evolution of the cluster (i.e., without any formation control) assuming ideal initial conditions.

A similar analysis has been conducted in [10], where a five-spacecraft formation was propagated numerically through GMAT while accounting for main low Earth orbit (LEO) perturbing effects (Earth gravity field, atmospheric drag, third body accelerations from Moon and Sun, and solar radiation pressure). Results can be summarized as follows:

- The difference in terms of ballistic coefficients among the platforms has to be minimized since differential drag is the primary reason for disruption of relative patterns;

- The precession of the arguments of perigee produces the deformation of the radial/crosstrack trajectories and thus a reduction of the minimum distance in the radial/crosstrack plane, particularly that of the deputy closest to the chief;

- The impact of the operational altitude (within the range 500-600 km) on spacecraft relative motion is not very significant when differential drag is minimized.

To give the reader some quantitative insight into the free dynamic evolution of the designed trajectories, an analysis was performed in GMAT by considering a formation made of a chief-transmitter and a deputy-receiver (the black-blue configuration shown in Figure 1). Satellites were assumed to be characterized by the same ballistic coefficient and to orbit at an altitude $(h)$ of $550 \mathrm{~km}$. Formation stability was investigated in both a low-inclination $\left(i=20^{\circ}\right)$ and a Sun-synchronous $\left(i=97.59^{\circ}\right)$ scenario. Additional details in terms of spacecraft features and orbit elements are provided in Table 1.

Figure 2 shows the results of orbit propagation for the low-inclined formation in terms of time variation of four main parameters: the radial/cross-track baseline $\left(d_{x z}\right)$, the radial 
component $(x)$ and cross-track component $(z)$ of the relative trajectory, and the argument of perigee of the reference orbit $(\omega)$.

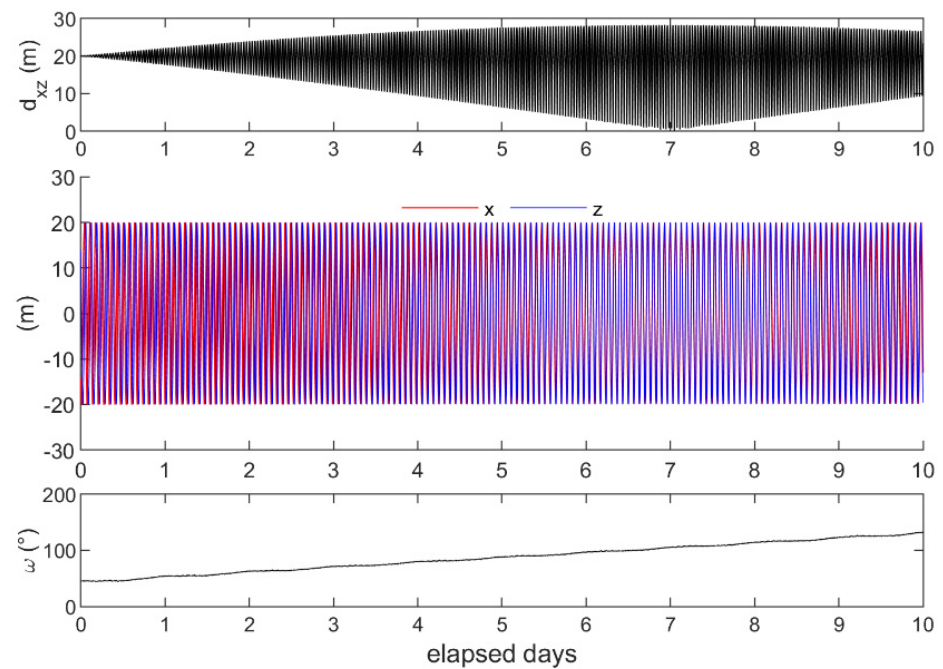

Figure 2. Two spacecraft formation based on safety tubes at low inclination: $h=550 \mathrm{~km}, d_{x z}=A_{x}=$ $A_{z}=20 \mathrm{~m}$. Evolution of $d_{x z}, x, z$, and $\omega$ for the first ten days of mission.

Since there is no observable drift of the $x$ and $z$ coordinates, the $d_{x z}$ deformation has to be attributed to the progressive phase shift between radial and cross-track oscillations. The latter is caused by the perigee's precession, which in turn mainly depends on absolute non sphericity (mostly J2) effects. An important feature that arises from this analysis is the non-divergence of the radial/cross-track baseline, which is closely related to the choice of imposing equal inclinations as a design constraint, thus preventing J2-induced differential nodal rates to be activated. Hence, a correct sizing of the $x$ and $z$ oscillations prevents the possibility of $d_{x z}$ exceeding any operational constraints dictated by the payload. On the contrary, the relative motion will in general diverge along the $y$ direction because of the alongtrack drift, that is only partially compensated when selecting orbits at equal altitudes [15].

Therefore, maintenance of safety tube-based geometries requires the deformation of the $d_{x z}$ baselines to be monitored, together with the along-track separation $y$ among the platforms.

The same analysis is repeated for a near-polar formation too (Figure 3). In such a scenario, the formation is stable for longer time periods because of the slower perigee precessions.
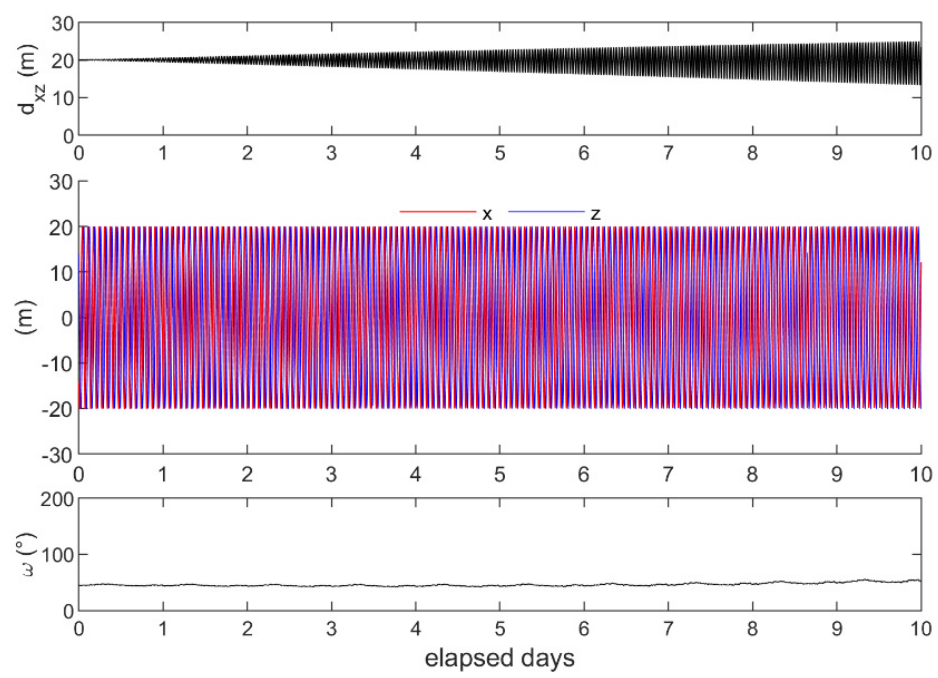

Figure 3. Two spacecraft Sun-synchronous formation based on safety tubes: $h=550 \mathrm{~km}, d_{x z}=A_{x}=$ $A_{z}=20 \mathrm{~m}$. Evolution of $d_{x z}, x, z$, and $\omega$ for the first ten days of mission. 
The effect of the inclination on formation stability is summarized in Table 2, where the time interval required for the radial/cross-track baseline $d_{x z}$ (nominally constant and equal to $20 \mathrm{~m}$ ) to violate a $10 \mathrm{~m}$ threshold is calculated for different operational altitudes (thus showing the negligible influence of this parameter). As for the along-track separation, the time required to exceed a $500 \mathrm{~m}$ threshold is estimated to be over 20 days for the two different inclinations.

Table 2. Comparison between number of days needed to violate a $d_{\text {min }}=10 \mathrm{~m}$ threshold for different altitudes.

\begin{tabular}{ccc}
\hline $\begin{array}{c}\mathbf{h} \\
\mathbf{( k m )}\end{array}$ & $\begin{array}{c}\text { Low-Inclination Orbit } \\
\text { (Days) }\end{array}$ & $\begin{array}{c}\text { Sun-Synchronous Orbit } \\
\text { (Days) }\end{array}$ \\
\hline 500 & 3.67 & 13.86 \\
525 & 3.75 & 14.07 \\
550 & 3.77 & 14.31 \\
575 & 3.86 & 14.52 \\
600 & 3.88 & 14.77 \\
\hline
\end{tabular}

\section{Formation Maintenance}

An adaptive strategy is proposed for maintenance of relative configurations. Indeed, every time some geometric conditions characterizing a specific formation are violated, a series of reconfiguration maneuvers are elaborated which are aimed at restoring nominal trajectories while accounting for the system's current state.

With reference to patterns based on safety tubes, control actions are necessary when:

- At least one of the imaginary tubes encompassing each satellite is trespassed;

- At least one of the along-track baselines exceeds a $Y_{M A X}$ threshold, that is assumed as the maximum separation for coherent processing of radar signals.

If one of the above-mentioned situations occurs, the cluster is reconfigured. In detail, the target geometry is computed according to the current state of a reference satellite of the formation. Assuming a reconfiguration time $t_{r}=K T$, where $K$ indicates an integer number and $T$ is the formation's orbit period, it is possible to propagate the chief's state for $t_{r}$ and to determine the new geometry related to the design set $\left[d_{x z}, A_{x}, A_{z}\right]$ through software that replicates the steps defined in Algorithm 1.

With the cluster's current state known and the desired one to be reached within $t_{r}$, the control actions required to transfer each deputy from its initial state $\left(^{0}\right)$ to the final one $\left(^{F}\right)$ have to be retrieved in terms of burn location and magnitude.

It is worth underlining that the key concept of relative motion control is that the formation is adaptively redesigned following the dynamic evolution of its chief. In general, this implies that the required differences in mean orbital parameters will also change in time. Furthermore, as the trajectory design process does not require any special constraint for the chief orbital parameters, this means that effects such as perigee precession are not counteracted but are accounted for in the maintenance process.

\subsection{Determination of Control Actions}

Each spacecraft is assumed to be equipped with a propulsive system able to provide a thrust along every main direction of its Hill reference frame. In order to determine the intensity and the location of each burn, the impulsive approach described in [9] is considered, that is conceived for fuel-optimal reconfiguration of formations on near-circular orbits. More specifically, the three-impulse strategy is implemented. Two impulses are used for variation of the in-plane components of relative motion $(i p)$ and the other one is exploited for modification of the out-of-plane baseline (oop).

Such a technique enables the $\Delta v$-s and the set $\left(u_{i p}^{1}, u_{i p}^{2}, u_{o o p}\right)$-whose elements identify the arguments of latitude for applications of the corresponding burns-to be determined in 
the Hill reference frame of each orbiting platform. Moreover, the formulation relies on the relative orbit elements:

$$
\delta o e_{j}=\left[\begin{array}{c}
\delta a \\
\delta \lambda \\
\delta e_{x} \\
\delta e_{y} \\
\delta i_{x} \\
\delta i_{y}
\end{array}\right]=\left[\begin{array}{c}
\left(a-a_{j}\right) / a_{j} \\
u-u_{j}+\left(\Omega-\Omega_{j}\right) \cos i_{j} \\
e \cos \omega-e_{j} \cos \omega_{j} \\
e \sin \omega-e_{j} \sin \omega_{j} \\
i-i_{j} \\
\left(\Omega-\Omega_{j}\right) \sin i_{j}
\end{array}\right]
$$

where the $j$ subscript identifies the $j$ th satellite in the formation (while no subscript is used to characterize the chief satellite) and the $\delta \lambda$ parameter is the relative mean longitude, defined as $\delta \lambda=\delta u+\delta i_{y} \cot i$. The employment of such a non-singular orbital set, especially of the $\delta \lambda$ variable instead of $\delta u$, allows the in-plane relative motion to be completely decoupled with respect to that occurring in the cross-track direction [9].

Hence, a maneuver that modifies the out-of-plane component of the relative trajectory entails a $\Delta$ variation of the relative orbit parameters $\delta i_{x}$ and $\delta i_{y}$. The change of these elements from the initial condition to the final one (i.e., $\Delta \delta i_{x}=\delta i_{x}^{F}-\delta i_{x}^{0}$ and $\Delta \delta i_{y}=\delta i_{x}^{F}-\delta i_{x}^{0}$ ) is produced by maneuvering the deputy $j$ at the argument of latitude $u_{o o p_{j}}$ with a $\Delta v_{z_{j}}$ so that [9]

$$
\begin{gathered}
u_{o o p_{j}}=\tan ^{-1}\left(\frac{\Delta \delta i_{y}}{\Delta \delta i_{x}}\right)+K \pi \\
\Delta v_{z_{j}}=v_{j} \sqrt{\Delta \delta i_{x}^{2}+\Delta \delta i_{y}^{2}}
\end{gathered}
$$

where $v$ represents the spacecraft velocity and $K$ is the integer number previously introduced to set the reconfiguration time.

The resolution of the in-plane reconfiguration problem from state 0 to state $F$, that for arbitrary values of $u_{i p_{j}}^{1}$ and $u_{i p_{j}}^{2}$ requires the solving of the linear system [9]

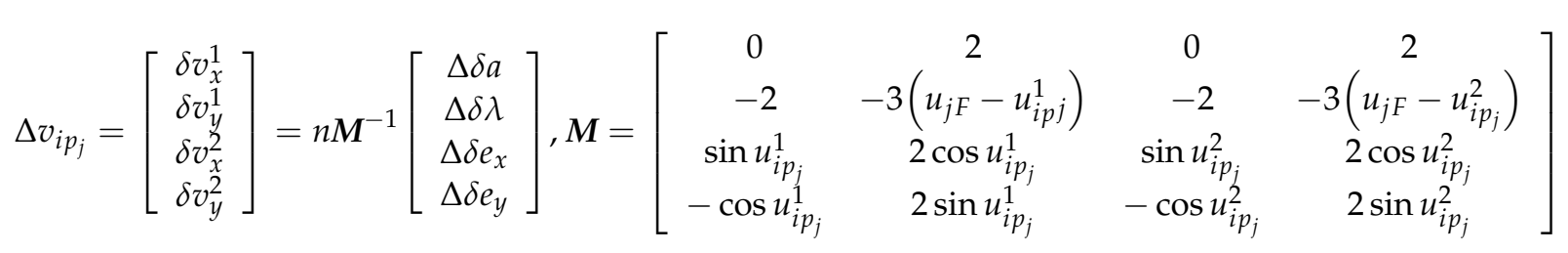

is handled by optimization software, that determines the values of $u_{i p_{j}}^{1}$ and $u_{i p_{j}}^{2}$ by minimizing the cost function $J=\Delta v_{i p_{j}}^{T} \Delta v_{i p_{j}}$ through resolution of the necessary conditions [9]

$$
\frac{\partial J}{\partial u_{i p_{j}}^{1}}=0 \quad \frac{\partial J}{\partial u_{i p_{j}}^{2}}=0
$$

In detail, the problem in Equation (4) is solved through a Newton-Raphson routine, whose initial guess corresponds to the cluster state at the beginning of the reconfiguration (the $n$ variable in Equation (3) represents the spacecraft mean angular motion).

\subsection{Considerations on System Organizational Architecture}

The space system is assumed to rely on a distributed architecture [16]. This means that each platform is considered to be provided with autonomous computational and data-handling capabilities, thus being able to determine its transfer orbit once the initial and final conditions are known. This requires the satellites to be connected on a local network for mutual data broadcasting, which should not pose significant technological challenges given the short baselines among the satellites and the limited data rate related to exchange of navigation information. Such a situation would allow every spacecraft to detect any deviation of the baselines from their nominal values, and hence to compute the new configuration through exploitation of an on-board algorithm devoted to formation design. 
In the case under consideration, the deputy satellite is meant to orbit without trespassing a safety tube around the chief, so that the $x z$ components of relative motion never fall below a threshold $d_{\text {min }}$. Moreover, the fractionated nature of the spaceborne SAR sets a limit to the maximum separation tolerable in the $y$ direction. An example of reconfiguration maneuvers for adaptive formation maintenance is shown in Figure 4, in case of both violation of cross-track constraints (on the left) and of extreme along-track separations (on the right).

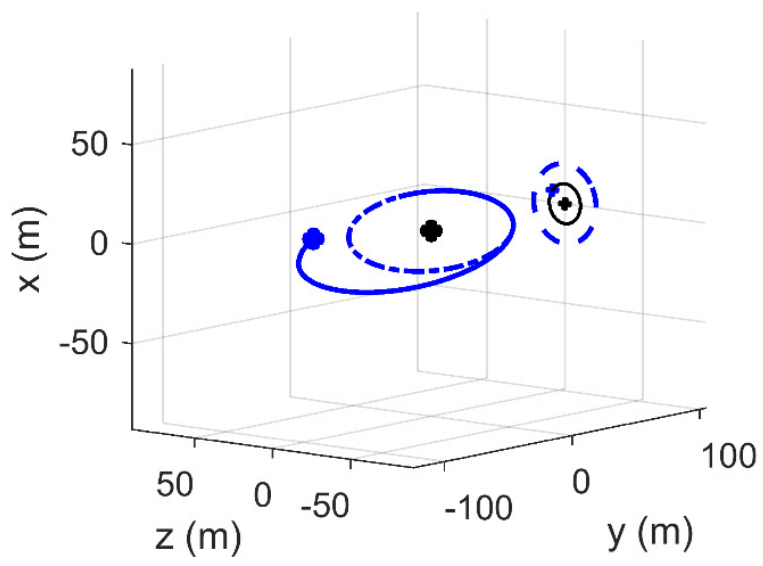

(a)

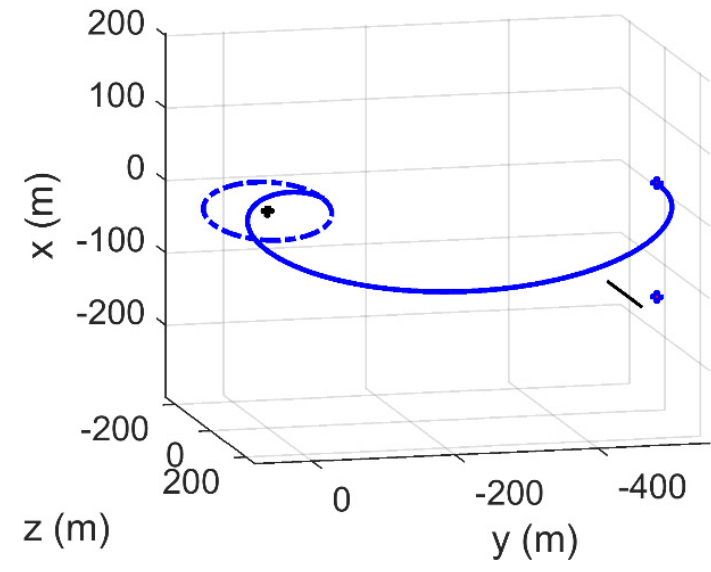

(b)

Figure 4. Maneuvers for formation maintenance. (a) The reconfiguration is triggered by the deputy trespassing a $10 \mathrm{~m}$ safety tube centered around the chief (projected in black solid lines on the $x z$ plane); (b) the reconfiguration is caused by the deputy exceeding a $500 \mathrm{~m}$ along-track separation (projected in black solid line on the $y z$ plane).

\section{Assessment of the Maintenance Strategy}

This section presents the results of an analysis performed using GMAT as orbit propagation software. Beyond proving the effectiveness of the adaptive procedure for maintenance of relative geometries, this section aims to provide an estimate of the control efforts required to preserve the spacecraft formation geometry. An overview of performance achievable in the presence of navigation errors and maneuvering execution errors is given too.

In the remaining part of this section, the focus is set on a two-spacecraft formation, with the chief considered as the fixed satellite and the deputy as the platform maneuvering around it. Considerations about the possibility to invert the roles, or about the need of a task-assignment strategy disciplining the reconfiguration of multi-spacecraft formations, are linked with ongoing research and are included in the conclusions.

\subsection{Simulation Environment}

The simulation environment relies on GMAT and MATLAB. In detail, GMAT was used to realistically propagate the spacecraft orbits in the presence of external perturbations. MATLAB was the programming language used for evaluation of target configurations (Algorithm 1) and generation of transfer trajectories (Equations (2)-(4)).

Figure 5 summarizes the general architecture of the simulator.

The system dynamics were entrusted to GMAT, which was exploited to calculate the formation relative geometry in the presence of external perturbations (Earth oblateness, aerodynamic drag, Sun radiation pressure, third body effects). The baselines were then processed in MATLAB to account for navigation errors. Relative navigation errors were assumed to be zero-mean white Gaussian variables with standard deviation $\sigma_{p}$ for position and $\sigma_{v}$ for velocity. 


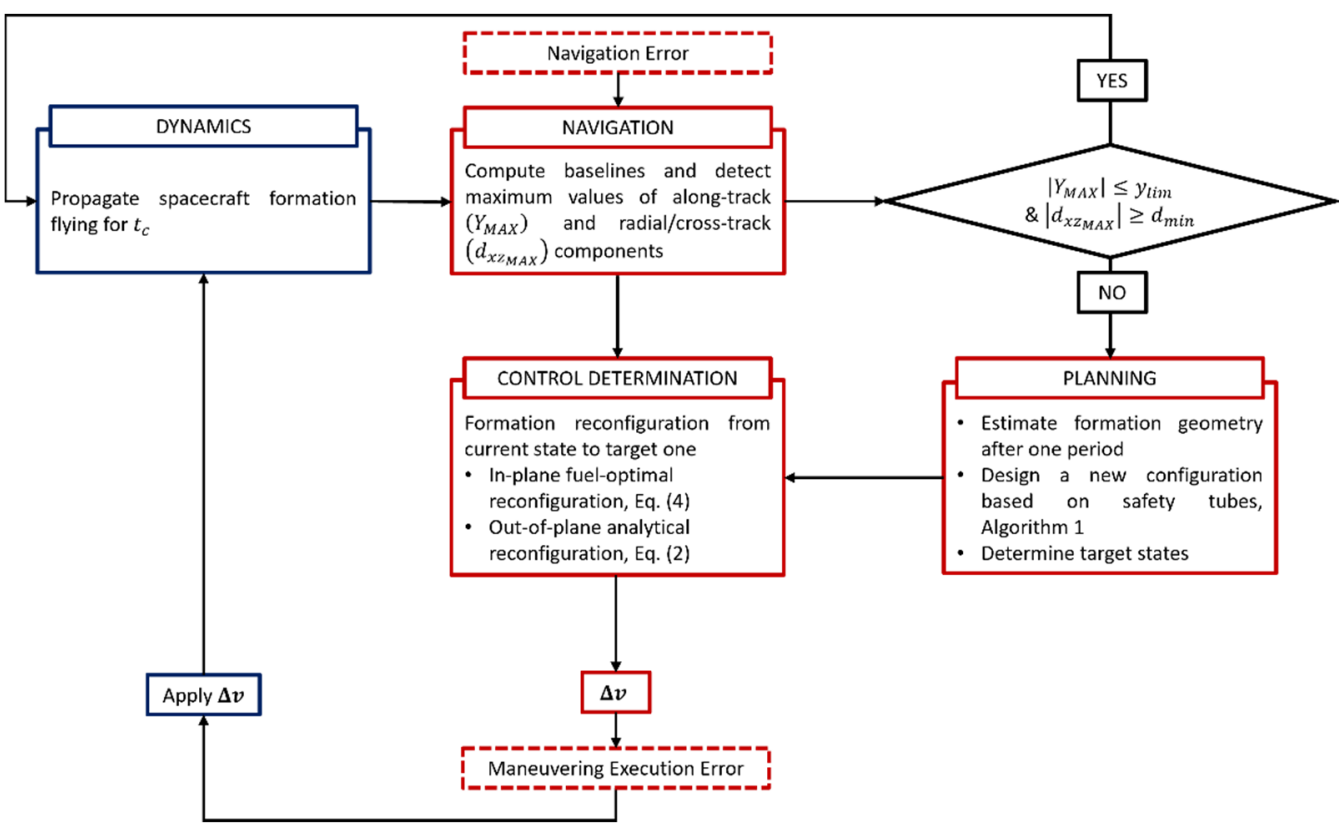

Figure 5. Architecture of the simulation environment realized through GMAT (blue boxes) and MATLAB (red boxes).

Hence, the cluster configuration was monitored as the spacecraft dynamics evolve. More specifically, the maximum cross-track baseline $\left(d_{x z_{M A X}}\right)$ was verified to not violate a threshold $d_{\min }$, while the maximum separation in the along-track $\left(Y_{M A X}\right)$ was checked to be always below $y_{\text {lim }}$. If both these conditions were satisfied, then the cluster motion was propagated in GMAT for a time $t_{c}$, which is the update time for the controller $\left(t_{c}=300 \mathrm{~s}\right.$ in considered simulations). On the contrary, the nonoccurrence of at least one of the above-mentioned circumstances triggers the planning and control determination sequence.

In detail, the planning algorithms were implemented in MATLAB to propagate the state of the chief satellite from its current condition $t_{0}$ till $t_{0}+t_{\text {rec }}$ (based on the current state, the chief was propagated using a Keplerian motion model complemented with the inclusion of secular J2 effects), where $t_{\text {rec }}$ represents the time required to complete the reconfiguration (in the considered scenario, $t_{\text {rec }}$ equals the chief's orbital period $T$ ). With the chief's predicted state available, a new configuration based on safety tubes can be designed through the pseudo-code described in Algorithm 1, which identifies the target pattern that has to be reached by specifying the differences in orbital parameters, and thus the set of relative orbit elements that the deputy should return to.

At this point, all the information required for computation of control actions was available. According to the methodology presented in Section 3.1, the control determination block is thus able to determine in the Hill reference frame of the deputy and the three impulses required for orbit maintenance, both in terms of magnitude $\left(\Delta v_{i p_{j}}, \Delta v_{z_{j}}\right)$ and location $\left(u_{i p_{j}}^{1}, u_{i p_{j},}^{2}, u_{o o p_{j}}\right)$. In particular, the best pair of in-plane maneuvers was derived through numerical optimization of Equation (4), while the out-of-plane burst was analytically computed from Equation (2).

The impulsive maneuver was thus executed in GMAT according to the outputs provided by MATLAB routines, which were conceived to potentially include maneuvering execution errors too.

\subsection{Performance Estimations}

Performance of the proposed maintenance strategy has been evaluated in terms of $\Delta v$ and maneuver frequency.

Simulations were performed by considering the spacecraft formation designed as in Section 2.2, with the reference satellite assumed in either a low-inclination (LI) or a 
Sun-synchronous (SS) orbit. The parameters that characterize the chief are always those specified in Table 1, except for the inclination, which is $20^{\circ}$ in the LI scenario. As for the formation design parameters, they were $d_{x z}=A_{x}=A_{z}=20 \mathrm{~m}$.

\subsubsection{Ideal Case}

Figure 6 shows the results relative to the first month of the mission. In such a circumstance, navigation errors are neglected, and the same considerations of Section 2.2 apply; that is, SS configurations are more stable than LI configurations. More specifically, the average period required for cluster reconfiguration is 3.88 days in the latter scenario (blue circular markers in Figure 6), while only 14.3 days occur between consecutive orbital corrections in the SS case (red square markers in Figure 6). The simulation results show that the mean $\Delta v$ per maneuver is $26.7 \mathrm{~mm} / \mathrm{s}$ in the LI scenario, with a total $\Delta v$ expenditure for the deputy satellite of $225 \mathrm{~mm} / \mathrm{s}$. The Sun-synchronous case requires a reduced control effort, with a total delta- $v$ of $34 \mathrm{~mm} / \mathrm{s}$ that corresponds to a mean $\Delta v$ per orbit of $17 \mathrm{~mm} / \mathrm{s}$.

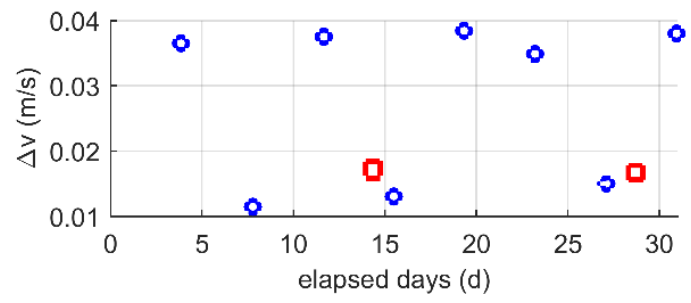

Figure 6. $\Delta v$ required for maintenance of safety tube-based formations over the first 30 days of mission $-h=550 \mathrm{~km}, d_{x z}=20 \mathrm{~m}$, no navigation errors. Blue circular markers refer to the LI scenario, while red square markers to the SS scenario.

A complete characterization of the maintenance strategy in the absence of navigation errors is provided in Table 3.

Table 3. Maneuvering frequency and $\Delta v$ required for maintenance of safety tube-based formations over the first 6 months of mission $-h=550 \mathrm{~km}$, no navigation errors.

\begin{tabular}{cccccc}
\hline Formation & $\begin{array}{c}\Delta v_{T O T} \\
(\mathbf{m} / \mathbf{s})\end{array}$ & $\begin{array}{c}\Delta v \text { Mean } \\
(\mathbf{m} / \mathbf{s})\end{array}$ & $\begin{array}{c}\Delta v \text { Std } \\
(\mathbf{m} / \mathbf{s})\end{array}$ & $\begin{array}{c}\text { Reconf. Time Mean } \\
(\mathbf{h})\end{array}$ & $\begin{array}{c}\text { Reconf. Time Std } \\
(\mathbf{h})\end{array}$ \\
\hline LI & 0.2248 & 0.0281 & 0.0124 & 92.8220 & 0.8882 \\
SS & 0.0340 & 0.0170 & 0.0005 & 344.4637 & 0.3023 \\
\hline
\end{tabular}

\subsubsection{Navigation Errors}

To preliminary evaluate the impact of navigation errors on formation maintenance, the same simulations were performed by adding to position and velocity measurements from GMAT zero-mean random errors characterized by a standard deviation on both position $\left(\sigma_{p}\right)$ and velocity $\left(\sigma_{v}\right)$. Concerning the orders of magnitudes for $\sigma_{p}$ and $\sigma_{v}$, these were selected in order to be consistent with the nominal performance of carrier phase differential GNSS techniques $[17,18]$.

Figure 7 shows the reconfigurations occurring over 1 month, assuming $\sigma_{p}=1 \mathrm{~cm}$ and different values of $\sigma_{v}(1$ and $5 \mathrm{~mm} / \mathrm{s}$, respectively).

As expected, the reconfiguration frequency increases. Moreover, the main reason for destruction of nominal geometries is the along-track drift among the spacecraft, whose occurrence is shown in Figure 7 by means of filled markers (the empty ones are used to identify reconfigurations triggered by alterations of the radial/cross-track baselines $\left.d_{x z}\right)$. Such an effect is predominant in both the scenarios, with relative percentages of $61 \%$ $\left(\sigma_{v}=1 \mathrm{~mm} / \mathrm{s}\right) / 64 \%\left(\sigma_{v}=5 \mathrm{~mm} / \mathrm{s}\right)$ in the low-inclination cases, and $86 \%\left(\sigma_{v}=1 \mathrm{~mm} / \mathrm{s}\right) /$ $54 \%\left(\sigma_{v}=5 \mathrm{~mm} / \mathrm{s}\right)$ in case of Sun-synchronous formations. 


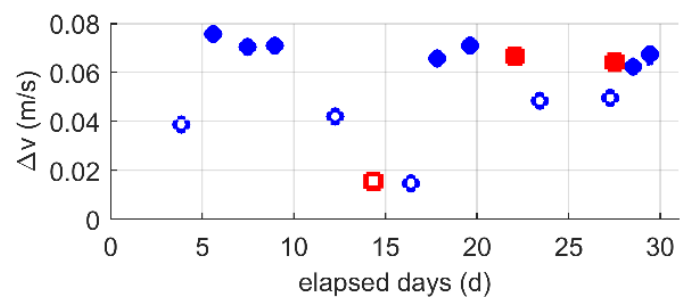

(a)

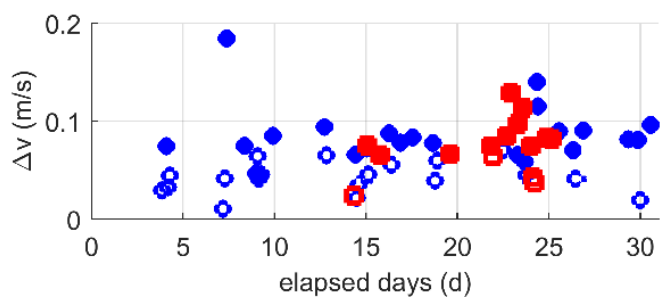

(b)

Figure 7. $\Delta v$ required for maintenance of safety tube-based formations over the first 30 days of mission $-h=550 \mathrm{~km}$. Navigation errors are: (a) $\sigma_{p}=1 \mathrm{~cm}, \sigma_{v}=1 \mathrm{~mm} / \mathrm{s} ;(\mathbf{b}) \sigma_{p}=1 \mathrm{~cm}, \sigma_{v}=5 \mathrm{~mm} / \mathrm{s}$. Blue circular markers refer to the LI scenario, while red square markers to the SS scenario. In addition, filled markers detect reconfigurations occurring because of $\left|Y_{M A X}\right| \geq 500 \mathrm{~m}$, while empty markers refer to maneuvers due to $\left|d_{x z_{M A X}}\right| \leq 10 \mathrm{~m}$.

A sensitivity analysis is presented and discussed in the remaining of this section. For each case - that is, for a given set of $\sigma_{p}$ and $\sigma_{v}$ values - results were derived by considering the formation evolution over 1 month and by running the simulation multiple times in order to obtain a significant amount of maneuvers. Then, statistics were derived.

In general, the presence of increasing navigation errors on spacecraft velocities is responsible for an erroneous reconstruction of the relative geometry. This entails an incorrect estimation of the separation among the satellites, and hence an increasing number of reconfiguration maneuvers. Since the nominal formation that must be reinstated is the same, the required delta- $v$ per maneuver is almost similar in both the LI and SS scenarios. Instead, the main differences arise when the total delta- $v$ expenditure $\left(\Delta v_{T O T}\right)$ and the time length between consecutive reconfigurations are considered.

As discussed in Section 2, low-inclined formations are characterized by a faster perigee precession with respect to the Sun-synchronous case because of Earth-induced perturbations. This causes a fast deformation of the $d_{x z}$ baseline and calls for a superior maintenance effort. Such an effect is further stressed in the presence of navigation errors. Figure 8a shows the result of multiple runs with $\sigma_{v}$ values of $0.5,1,2.5$, and $5 \mathrm{~mm} / \mathrm{s}$. Statistics have been then interpolated for trend reconstruction. In this case, the higher $\sigma_{v}$ is, the higher $\Delta v_{T O T}$ is in terms of both mean and standard deviations. Differently, the maneuvering period reduces in terms of mean values (from $80.9 \mathrm{~h}$ for $\sigma_{v}=0.5 \mathrm{~mm} / \mathrm{s}$ to $16.8 \mathrm{~h}$ for $\sigma_{v}=5 \mathrm{~mm} / \mathrm{s}$ ), but a similar trend cannot be found when considering standard deviations because of the higher formation instability. The main results of LI-related simulations are summarized in Table 4.
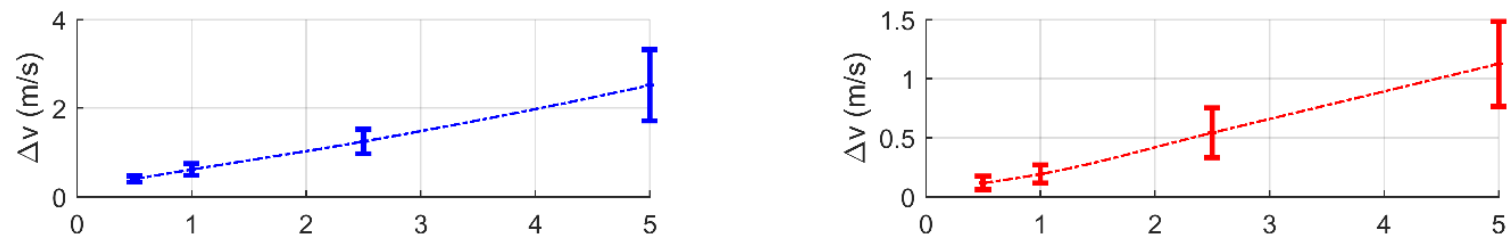

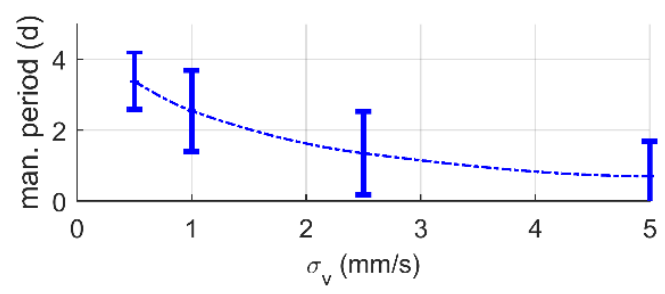

(a)

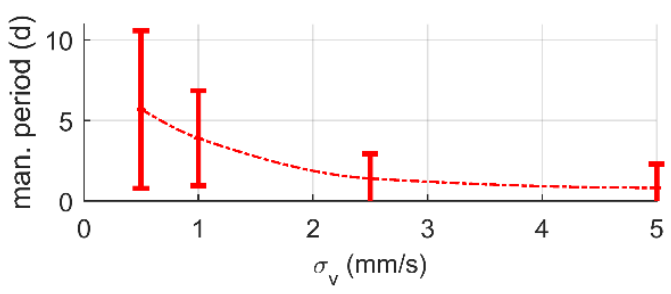

(b)

Figure 8. Sensitivity analyses for maintenance of low-inclined (a) and Sun-synchronous (b) formations in the presence of navigation errors $\left(\sigma_{p}=1 \mathrm{~cm}\right)$. Total $\Delta v$ and maneuvering period over the first 30 days of mission $-h=550 \mathrm{~km}$. 
Concerning Sun-synchronous formations, these were designed to account for J2 effects. Therefore, the total control effort is less than that required to maintain LI formations. As an example, the worst-case analyzed scenario (that is $\sigma_{v}=5 \mathrm{~mm} / \mathrm{s}$ ) is characterized almost by half the $\Delta v_{\text {TOT }}$ required in the LI case $(1.12 \mathrm{~m} / \mathrm{s}$ with respect to $2.52 \mathrm{~m} / \mathrm{s})$. This happens because of the less frequent maneuvering periods needed when exploiting orbits at high inclinations, which in turn are less affected by perigee precession. These conclusions can be derived looking at the trends in Figure $8 \mathrm{~b}$. Here, the main difference with respect to Figure 8a concerns the maneuvering period, that decreases for higher $\sigma_{v}$ values both in terms of mean and standard deviations. The statistics relative to the SS configurations are summarized in Table 5.

Table 4. Statistics related to the sensitivity analyses for maintenance of low-inclined formations in the presence navigation errors $\left(\sigma_{p}=1 \mathrm{~cm}\right)$.

\begin{tabular}{ccccccc}
\hline $\begin{array}{c}\sigma_{v} \\
(\mathbf{m m} / \mathbf{s})\end{array}$ & $\begin{array}{c}\Delta v_{T O T} \\
\text { Mean }(\mathbf{m} / \mathbf{s})\end{array}$ & $\begin{array}{c}\Delta v_{T O T} \\
\text { Std }(\mathbf{m} / \mathbf{s})\end{array}$ & $\begin{array}{c}\Delta v \\
\text { Mean }(\mathbf{m} / \mathbf{s})\end{array}$ & $\begin{array}{c}\Delta v \\
\text { Std }(\mathbf{m} / \mathbf{s})\end{array}$ & $\begin{array}{c}\text { Reconf. Time Mean } \\
(\mathbf{h})\end{array}$ & $\begin{array}{c}\text { Reconf. Time Std } \\
(\mathbf{h})\end{array}$ \\
\hline 0.5 & 0.400 & 0.070 & 0.049 & 0.018 & 80.957 & 18.867 \\
1 & 0.614 & 0.129 & 0.058 & 0.018 & 61.062 & 27.426 \\
2.5 & 1.247 & 0.276 & 0.063 & 0.019 & 32.378 & 28.329 \\
5 & 2.516 & 0.802 & 0.069 & 0.028 & 16.832 & 23.507 \\
\hline
\end{tabular}

Table 5. Statistics related to the sensitivity analyses for maintenance of Sun-synchronous formations in the presence navigation errors $\left(\sigma_{p}=1 \mathrm{~cm}\right)$.

\begin{tabular}{ccccccc}
\hline $\begin{array}{c}\sigma_{v} \\
(\mathbf{m m} / \mathbf{s})\end{array}$ & $\begin{array}{c}\Delta v_{T O T} \\
\text { Mean }(\mathbf{m} / \mathbf{s})\end{array}$ & $\begin{array}{c}\Delta v_{T O T} \\
\text { Std }(\mathbf{m} / \mathbf{s})\end{array}$ & $\begin{array}{c}\Delta v \\
\text { Mean }(\mathbf{m} / \mathbf{s})\end{array}$ & $\begin{array}{c}\Delta v \\
\text { Std }(\mathbf{m} / \mathbf{s})\end{array}$ & $\begin{array}{c}\text { Reconf. Time Mean } \\
(\mathbf{h})\end{array}$ & $\begin{array}{c}\text { Reconf. Time Std } \\
(\mathbf{h})\end{array}$ \\
\hline 0.5 & 0.117 & 0.056 & 0.044 & 0.022 & 136.594 & 117.525 \\
1 & 0.191 & 0.077 & 0.050 & 0.021 & 93.506 & 70.831 \\
2.5 & 0.541 & 0.209 & 0.058 & 0.019 & 33.657 & 36.573 \\
5 & 1.125 & 0.359 & 0.066 & 0.027 & 19.809 & 35.336 \\
\hline
\end{tabular}

\subsubsection{Maneuvering Execution Errors}

In nominal conditions, maintenance maneuvers do not constitute a threat for formation safety. Indeed, the natural effect of the maneuvers is to increase the radial/cross-track separation. When erroneous delta-v are applied, instead, some risks are generated due to the short distance between the spacecraft. While analysis of reactive closed-loop strategies is certainly a very important point to be addressed in future works, this section is meant to further assess the impacts of a non-nominal operational environment on delta- $v$ budget.

The followed approach is similar to the one described in Section 4.2.2: besides navigation errors, several simulations were performed by adding to each burst a zero-mean random error with a standard deviation that is a percentage of its magnitude. The formation evolution was monitored over a month, and multiple runs were considered to obtain a significant number of maneuvers and derive statistics. The results of such a sensitivity analysis are reported in Figure 9, where the blue and red trends show the control effort required for maintenance of LI and SS formations. In this case, navigations errors are assumed to be $\sigma_{p}=1 \mathrm{~cm}$ and $\sigma_{v}=2.5 \mathrm{~mm} / \mathrm{s}$.

In general, due to the thruster errors, the desired configuration cannot be fully restored. Consequently, the relative geometry degrades faster, and the number of orbital maneuvers increases.

Results can be summarized as follows: for moderate execution errors (5\%), mean values are comparable with those reported in Tables 4 and 5 (lines with $\sigma_{p}=2.5 \mathrm{~mm} / \mathrm{s}$ ), and thus underlining that navigation errors are still driving the budget. If the thruster accuracy becomes worse, this error source takes over navigation errors. In particular, the estimated $\Delta v_{T O T}$ values double for burst errors up to $25 \%(2.48 \mathrm{~m} / \mathrm{s}$ in the LI case and $1.12 \mathrm{~m} / \mathrm{s}$ in the SS case), while mean maneuvering times approximately halve (16.5 
and $19.6 \mathrm{~h}$ for the LI and SS configurations, respectively). Further details can be found in Tables 6 and 7.

Assuming the same boundary conditions, the analyses were then repeated by considering different ballistic coefficients between the satellites. Indeed, even if the system is designed to minimize any deviation of these parameters from a reference value, a more realistic operational scenario should account for potential differences due to other mission constraints (attitude variation along the orbit, mass reduction, etc.).

The derived results are represented in Figure 9 with dashed lines (light blue for the LI case and yellow for the SS case). They refer to a framework that accounts for a 10\% difference between the ballistic coefficients. Under such a circumstance, the differential drag contributes to distortion of nominal patterns to a greater extent. Moreover, since the planning algorithms are based on a dynamic model that does not take this perturbation into account, the computed delta- $v$ are not accurate enough and maneuvers occur more frequently due to the short satellite distances. The main outcome is that, with equal perturbing sources, the resulting $\Delta v_{T O T}$ and maneuvering period are very similar, whatever the orbital inclination. For the sake of clarity, the statistics shown in Figure 9 are reported in Tables 6 and 7.
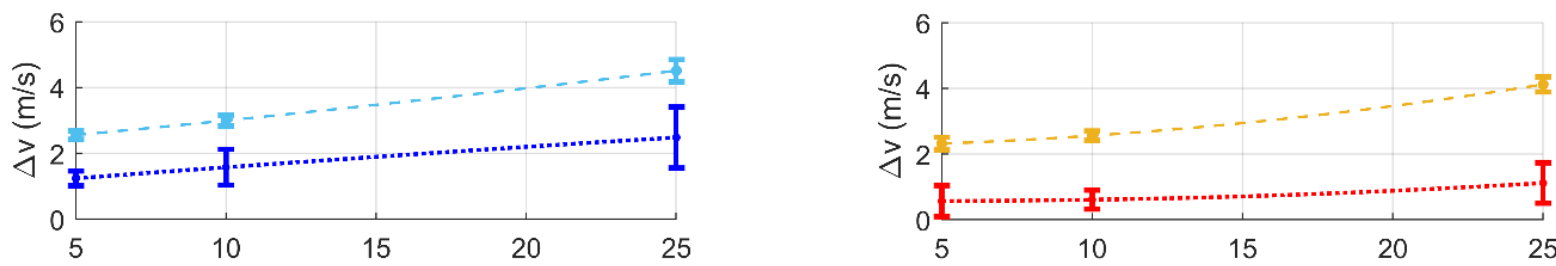

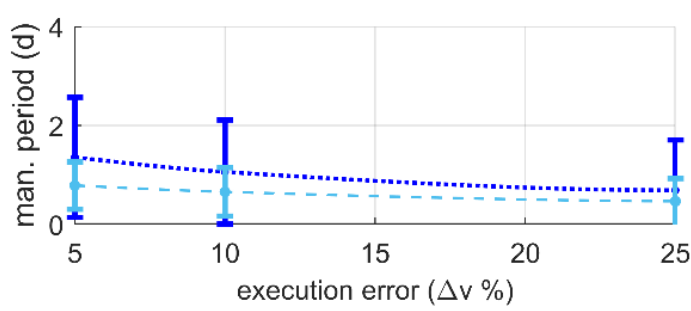

(a)

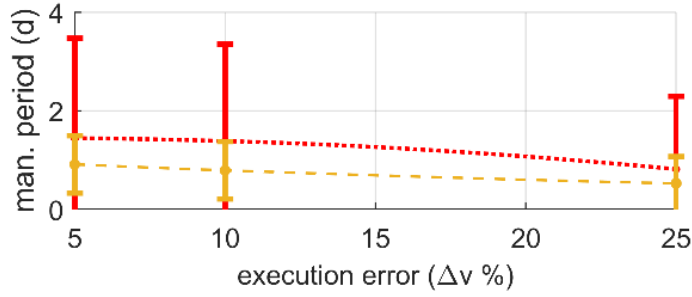

(b)

Figure 9. Sensitivity analyses for maintenance of low-inclined (a) and Sun-synchronous (b) formations in the presence of maneuvering execution errors and navigation errors $\left(\sigma_{p}=1 \mathrm{~cm}, \sigma_{p}=2.5 \mathrm{~mm} / \mathrm{s}\right)$. Total $\Delta v$ and maneuvering period over the first 30 days of mission $-h=550 \mathrm{~km}$. Differently from the dotted lines, the dashed lines account for a $10 \%$ difference between ballistic coefficients.

Table 6. Statistics related to the sensitivity analyses for maintenance of low-inclined formations in the presence navigation errors $\left(\sigma_{p}=1 \mathrm{~cm}, \sigma_{v}=2.5 \mathrm{~mm} / \mathrm{s}\right)$, maneuvering execution error, and ballistic coefficient differences.

\begin{tabular}{cccccccc}
\hline $\begin{array}{c}\text { Execution } \\
\text { Error } \\
(\boldsymbol{\Delta} \boldsymbol{v})\end{array}$ & $\begin{array}{c}\text { Ballistic } \\
\text { Coeff. Diff. } \\
(\mathbf{\%})\end{array}$ & $\begin{array}{c}\Delta v_{T O T} \text { Mean } \\
(\mathbf{m} / \mathbf{s})\end{array}$ & $\begin{array}{c}\Delta v_{T O T} \text { Std } \\
(\mathbf{m} / \mathbf{s})\end{array}$ & $\begin{array}{c}\Delta v \text { Mean } \\
(\mathbf{m} / \mathbf{s})\end{array}$ & $\begin{array}{c}\Delta v \text { Std } \\
(\mathbf{m} / \mathbf{s})\end{array}$ & $\begin{array}{c}\text { Reconf. } \\
\text { Time Mean } \\
(\mathbf{h})\end{array}$ & $\begin{array}{c}\text { Reconf. } \\
\text { Time Std } \\
(\mathbf{h})\end{array}$ \\
\hline 5 & 0 & 1.238 & 0.220 & 0.063 & 0.020 & 32.526 & 29.275 \\
5 & 10 & 2.555 & 0.131 & 0.069 & 0.025 & 18.802 & 11.546 \\
10 & 0 & 1.576 & 0.546 & 0.064 & 0.023 & 25.385 & 25.347 \\
10 & 10 & 2.996 & 0.165 & 0.067 & 0.027 & 15.702 & 11.803 \\
25 & 0 & 2.482 & 0.928 & 0.065 & 0.030 & 16.497 & 24.428 \\
25 & 10 & 4.511 & 0.340 & 0.073 & 0.036 & 11.166 & 11.191 \\
\hline
\end{tabular}


Table 7. Statistics related to the sensitivity analyses for maintenance of Sun-synchronous formations in the presence navigation errors $\left(\sigma_{p}=1 \mathrm{~cm}, \sigma_{v}=2.5 \mathrm{~mm} / \mathrm{s}\right)$, maneuvering execution error, and ballistic coefficient differences.

\begin{tabular}{|c|c|c|c|c|c|c|c|}
\hline $\begin{array}{c}\text { Execution } \\
\text { Error } \\
(\Delta v \%)\end{array}$ & $\begin{array}{c}\text { Ballistic } \\
\text { Coeff. Diff. } \\
(\%)\end{array}$ & $\begin{array}{c}\Delta v_{T O T} \text { Mean } \\
(\mathrm{m} / \mathrm{s})\end{array}$ & $\begin{array}{c}\Delta v_{T O T} \mathrm{Std} \\
\quad(\mathrm{m} / \mathrm{s})\end{array}$ & $\begin{array}{c}\Delta v \text { Mean } \\
(\mathrm{m} / \mathrm{s})\end{array}$ & $\begin{array}{c}\Delta v \text { Std } \\
(\mathrm{m} / \mathrm{s})\end{array}$ & $\begin{array}{c}\text { Reconf. } \\
\text { Time Mean } \\
\text { (h) }\end{array}$ & $\begin{array}{c}\text { Reconf. } \\
\text { Time Std } \\
\text { (h) }\end{array}$ \\
\hline 5 & 0 & 0.568 & 0.476 & 0.060 & 0.020 & 34.692 & 48.670 \\
\hline 5 & 10 & 2.317 & 0.191 & 0.072 & 0.018 & 21.873 & 13.945 \\
\hline 10 & 0 & 0.610 & 0.290 & 0.059 & 0.023 & 33.246 & 47.201 \\
\hline 10 & 10 & 2.560 & 0.144 & 0.070 & 0.021 & 18.979 & 13.943 \\
\hline 25 & 0 & 1.117 & 0.616 & 0.066 & 0.031 & 19.560 & 35.413 \\
\hline 25 & 10 & 4.118 & 0.229 & 0.076 & 0.034 & 12.635 & 13.071 \\
\hline
\end{tabular}

\section{Conclusions}

In this paper, a strategy for formation maintenance was proposed, with emphasis on the two-satellites case. The main aim was to preserve the formation flight of spacecraft orbiting very close to each other in order to realize a distributed SAR.

An adaptive strategy was proposed that sets the arrival pattern according to the expected state of a chief satellite at the end of the maneuver. Orbital corrections only affect the deputy, which moves around such a reference platform till reaching the configuration defined by a formation design algorithm based on safety tubes.

The developed approach is effective in preserving such a kind of geometries.

In absence of navigation errors, the baseline deformation in the $x z$ plane (mainly induced by perigee precession) is the primary reason of reconfiguration. The maneuvering frequency is estimated around 4 days for LI orbits, contrary to the 15 days required when considering SS cases. The total $\Delta v$ per maneuver is of some cm/s.

Inclusion of moderate relative navigation errors allows a more realistic estimation of control efforts. Under these circumstances, the maintenance is mostly triggered by an excessive separation between the platforms in the $y$ direction-especially for the SS scenario, where it is responsible for almost the entirety of reconfigurations. Therefore, the maneuvering frequency increases, while the $\Delta v$-s per maneuver are always of the order of several $\mathrm{cm} / \mathrm{s}$ and a moderate increase can be detected. The main difference is in the total delta-v, which is higher in the LI scenarios owing to a superior formation instability. In the analyzed worst-case frameworks, the $\Delta v_{T O T}$ for maintenance are estimated to be of the order of $2.5 \mathrm{~m} / \mathrm{s}$ per month (LI case) and of $1 \mathrm{~m} / \mathrm{s}$ per month (SS case).

Such a behavior is further emphasized when adding maneuvering execution errors, which become relevant for magnitudes above $5 \%$ of the delta- $v$ required from each burst. A similar effect is induced by the presence of different ballistic coefficients: these introduce differential drag perturbations that are not considered by the path-planning models. The result is a less accurate delta- $v$, and hence an increased maneuver frequency (regardless of the orbital inclination).

As a general conclusion, while the stability of the designed trajectories and the limited requirements of the distributed payload allow a reduced control effort, relative navigation errors play a major role, which is emphasized by the tight separation among spacecraft. This tight separation also impacts the control requirements in terms of accuracy, and the safety concerns in case of non-nominal thrust application or failures. These aspects encourage the adoption of closed-loop control strategies and low thrust solutions such as electric propulsion. It is also clear that allowing a larger radial/cross-track separation in DSAR operations is the primary way to relax relative navigation requirements, reduce safety concerns, and guarantee a prolonged remote sensing activity (that on the other hand would be interrupted by continuous reconfiguration maneuvers). Future research will be aimed at further investigating the impact of relative navigation errors and at implementing low thrust control strategies for adaptive formation maintenance, based on both open- and closed-loop approaches. In view of multi-satellite architectures comprising a chief and more 
than a single deputy, a task assignment logic will be also defined, as to provide the DSAR formation with enhanced autonomy. Moreover, innovative solutions will be investigated aimed at optimizing the total propellant consumption. The main emphasis will be given to strategies conceived to modify the role of the chief within a formation. In this way, the propellant for orbital correction can be redistributed among the component satellites. This will ensure a global mass balancing and reduce the difference among ballistic coefficients over time.

Author Contributions: Writing—original draft, S.S., M.I. and G.F. All authors have read and agreed to the published version of the manuscript.

Funding: This research received no external funding.

Institutional Review Board Statement: Not applicable.

Informed Consent Statement: Not applicable.

Data Availability Statement: Data is contained within the article.

Conflicts of Interest: The authors declare no conflict of interest.

\section{References}

1. D'Errico, M. Distributed Space Missions for Earth System Monitoring; Springer Science \& Business Media: Berlin, Germany, 2013; ISBN 9781461445418.

2. $\quad$ Bentum, M.; Verma, M.; Rajan, R.; Boonstra, A.; Verhoeven, C.; Gill, E.; van der Veen, A.; Falcke, H.; Wolt, M.K.; Monna, B.; et al. A roadmap towards a space-based radio telescope for ultra-low frequency radio astronomy. Adv. Space Res. 2020, 65, 856-867. [CrossRef]

3. Mittermayer, J.; López-Dekker, P.; Kraus, T.; Krieger, G. Small satellite dispersed SAR-an exemplary configuration. In Proceedings of the EUSAR 2016: 11th European Conference on Synthetic Aperture Radar, Hamburg, Germany, 6-9 June 2016.

4. Graziano, M.D.; Renga, A.; Grasso, M.; Moccia, A. PRF Selection in Formation-Flying SAR: Experimental Verification on Sentinel-1 Monostatic Repeat-Pass Data. Remote Sens. 2019, 12, 29. [CrossRef]

5. Moccia, A. Synthetic Aperture Radar. In Encyclopedia of Aerospace Engineering; John, W. \& Sons, Ltd.: Hoboken, NJ, USA, 2012; ISBN 9780470686652.

6. Rosen, P.A.; Hensley, S.; Joughin, I.R.; Li, F.K.; Madsen, S.N.; Rodriguez, E.; Goldstein, R.M. Synthetic aperture radar interferometry. Proc. IEEE 2000, 88, 333-380. [CrossRef]

7. Fasano, G.; D'Errico, M. Modeling orbital relative motion to enable formation design from application requirements. Celest. Mech. Dyn. Astron. 2009, 105, 113-139. [CrossRef]

8. Opromolla, R.; Fasano, G.; Rufino, G.; Grassi, M. Design of relative trajectories for in orbit proximity operations. Acta Astronaut 2018, 145, 342-356. [CrossRef]

9. Gaias, G.; D'Amico, S. Impulsive Maneuvers for Formation Reconfiguration Using Relative Orbital Elements. J. Guid. Control Dyn. 2015, 38, 1036-1049. [CrossRef]

10. Fasano, G.; Grassi, M.; Graziano, M.D.; Moccia, A.; Opromolla, R.; Renga, A.; Rufino, G.; Iervolino, M.; Sarno, S. Small Satellite Formation Flying for Distributed Synthetic Aperture Radar. Proc. Int. Astronaut. Congr. 2022, 192, 97-112.

11. Villano, M.; Krieger, G.; Moreira, A. Staggered SAR: High-Resolution Wide-Swath Imaging by Continuous PRI Variation. IEEE Trans. Geosci. Remote Sens. 2014, 52, 4462-4479. [CrossRef]

12. Chapin, E.; Chen, C. Along-track interferometry for ground moving target indication. IEEE Aerosp. Electron. Syst. Mag. 2008, 23, 19-24. [CrossRef]

13. Fornaro, G.; Pascazio, V.; Schirinzi, G. Resolution improvement via multipass SAR imaging. In Proceedings of the International Geoscience and Remote Sensing Symposium (IGARSS), Sydney, Australia, 9-13 July 2001.

14. Kraus, T.; Krieger, G.; Moreira, A.; Bachmann, M. Addressing the Terrain Topography in Distributed SAR Imaging. In Proceedings of the IET International Conference on Radar Systems (Radar), Toulon, France, 23-27 September 2019.

15. Alfriend, K.; Vadali, S.R.; Gurfil, P.; How, J.; Breger, L. Spacecraft Formation Flying: Dynamics, Control and Navigation; Elsevier Astrodynamics Series; Elsevier Science: Amsterdam, The Netherlands, 2009; ISBN 9780080559650.

16. Araguz, C.; Bou-Balust, E.; Alarcón, E. Applying autonomy to distributed satellite systems: Trends, challenges, and future prospects. Syst. Eng. 2018, 21, 401-416. [CrossRef]

17. Montenbruck, O.; D'Amico, S. GPS Based Relative Navigation. In Distributed Space Missions for Earth System Monitoring; Springer Science and Business Media LLC: Berlin, Germany, 2012; ISBN 9781461445418.

18. Graziano, M.D.; Renga, A.; Grasso, M.; Moccia, A. Error sources and sensitivity analysis in formation flying synthetic aperture radar. Acta Astronaut. 2021, 192, 97-112. [CrossRef] 